Rev. Chil. Pediatr. 68 (5); 216-219, 1997

\title{
Medición continua de presión intracraneana en niños
}

\author{
Cristina Pierry V.' ; Jorge Rnque E. ${ }^{2}$; Mario Cerda S. ${ }^{2}$; Silvia Arriagada T. ${ }^{2}$; Alejandro Donoso F. ${ }^{2}$; \\ Gabriel Muñoz R. ${ }^{2}$; Rafael Torres N. ${ }^{2}$; Guillermo Uslar G. ${ }^{2}$
}

\begin{abstract}
Resumen
Se describe una cucitaria clínica de a medición conlinua de a pesicn intracraneana a largo ce cuatro años en una unidr.d ce cuidados intensivos ped ćtr.co de un hosplal privado de Sontiago, Chile, de la información recistrada en las hisiorias clinicas de 21 n.ños (1 4 varones) de un mes a 15 años de edod, en que ei método fue empleado. Las entermedades $p$ incizales erari tumor cerebral $\{n=7 \mid$, 'rour arismo cronecencefático $(n=5)$. maltormaciones ciel sistema nerviosc central $\{n=4\}$, astix a zor i-mersion $\{n=3 \mid$ y menıngitis bacterına aguda $\{n=2\}$. Les rozones pcra indicar el pracedimien:o fueron hidrocetel:o agudo in= 13 i. contusión cerebrol mültiple (n=5; y edemo cerebral difuso in=3). En todos los cosos se realizó torografio axicl comoutadorizoda cerebral antes y después de instalor los calé-e:es y examen culoguirrica y cútivo diarios de liquido cerebroespnat. a h perlensión inracraneana ipresión mayor que 15 mmi de $\mathrm{Hg}$ l se reg stro en 19 casos que fueron ratodos con combinaciones de hipervent loción. monitol y riopenlal.

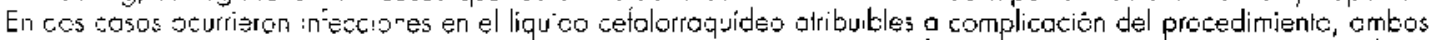
responcieron sié a los antibiót cos intratecales. E- un paciente ourró una hemorragia intraparenouimorosa cerebra! Dos pacientes con asfixia sor inmersion y uno con TEC grave y contusiones cerebrales múltiples, lodos ccn hipertensión impracraneano y déficit de presión de perfusiór cerebra', fallecteron. En dos 'a baja presión de perfusión se cebia o la hipenensićn nracraneana, en el eicero a céficíl de prosión arieral medio. Diecisiele pccientes egresaron en buerios condic ones y uno se rasladó con Glasgow 9 a olro servicio. as mediciones de presión introcraneano fueron confiables y fazilitaron la loma correcta de decisiones.
\end{abstract}

(Polabras clave: presión inilrocraneana, hiperlensión iniracianearie, niños.)

\section{Intracranial pressure monitoring in children}

Opiective: to dexcribe indicotions and comolicalions $0^{\circ}$ intracionial pressure monitoring in children in a privale clinic setting Methods: clinica eccrds of 21 patients $2 \angle$ maet aged one month lo 15 years, submited ro continuous initacionial pressure monitorig were reviewed for evidence $\sigma^{2}$ intracranial hifertension, and complications of the procedure. Resuits: tasic dicgrcsis were cerebral tumcr $(r:=7 !$, read injury with cronicancephalic trauma (n=5), central nervols syetem malformations $(n=4$, , near drowning $(n=3)$ and maningitis $i n=2)$. Inracranial pressure monitoring was indicaled by cocue hydruceptralus $n=73$ ], multiple cerebra! contusions and intracerebral hemoritages $\{r=5$ p and diffuse cerebral edema $(n=3)$. In 19 palienls introcranial hypetension ipressure goater than 15 mm $\mathrm{Hgl}$ was recorded Cerebrospincl fluid infection occurred in two paterits and porenchymal bleeding in che cose. Infections were stccestuf $y$ reated with intrcthecol aritibiotics. Two palients with near drowning and one with cerebral injury cnd riultiple ceretiral ccnlus on died, all of then had iril:acranial hyperension and abnormally low cerebral perfusion pressures. Seventeen pelienls were dischargac in good heolth and one was ransferred to another ce-ler with o 9 Glosjow sccre. Conctusicn: ir all coses, monitoring allowed appropriaie measurements of intracranial pressure and vecision making.

(Key words: intrccron ol sressure, roni•cring, children.)

1. Becado. [Pepartamento de Pediatría y Cirugía Infanti] Occidente, Facultad de Medicina Universidad de Chile.

2. Únidad de Tratanicusto Intensivo Pediátrica. Clínica Alemana de Santiago.
La medición continua de la presión intracraneana (PIC) por método invasivo es útil para manejar pacientes con afecciones neurológicas en que hay riesgo de hipertensión intracraneana 
(HIC) ${ }^{1.2}$, pues pormite detectar precozmente sus fluctuaciones y aplicar tratamientos antes que ocurran lesiones irreversibles en el sistema nervioso central (SNC) ${ }^{3-5}$, ya que la HIC y su intensidad no guardan estricta relación con los signos neurológicos clínicos que, por lo demás, sufren modificaciones con los sedantes y paralizantes que se emplean frecuentemente en estos pacientes, dificultando aứn más su interprelación ${ }^{2,6-8}$. Tan importante como la medición y control de la PIC es preservar la normalidad de la presión de perfusión cerebral (PPC), que se calcula por la diferencia entre PIC y presión arterial media (PAM) y es un indicador de ta calidad de la irrigación del tejido nervioso. Por esta razón la presión arterial baja es tan deletérea en estos casos, como la presión intracraneara desusadamente alta.

Aunque la medición continua de la PIC es relativamente poco usada en Chile por limitaciones de recursos, en otros lugares se incluye en Ios estándares de tratamiento de pacientes con traumatismo encefalocraneano (TEC) grave fcontusiones cerebrales importantes y posoperatorio inmediato de contusiones hemorrágicas $)^{2.9}$; bloqueo del sistema de circulación del líquido cefalorraquídeo (LCR) por tumores o infecciones del SNC, antes de la instalación de válvulas de derivación; y también en algunos casos de edema cerebral difuso, como encefalopatías hipóxico isquémicas o metabólicas.

Existen dos tipos de catéteres para medir PIC: los de polietileno-que transmiten la presión a través de una columna de solución de $\mathrm{NaCl} 0,9 \%$ desde alguno de los compartimientos cerebrales-y los de fibra óptica, en que la información se envía en forma de pulsos luminosos desde el sitio de registro al instrumento que los lee en términos de ondas de presión en los sistemas de medición. Este último dispositjvo permite registros más precisos, pues no necesita que el sistema esté permeable para funcionar, razón por la cual es el más recomendado, pese a su mayor coste ${ }^{3}$. Los llamados captores (captadores) subdurales no son más que una modificación de los catéteres de polietileno, cuya forma les permite adaptarse con mayor facilidad a dicho espacio.

Ambos sistemas pueden instalarse en ubicaciones extradural o intraventricular y el de fibra óptica en el parénquima cerebral. La ubicación intraventricular, cuando es posible, es la preferi- da. pues también permite drenar LCR para disminuir la $\mathrm{HIC}^{3-6}$. para lo cual los catéteres de fibra óptica intraventriculares cuentan con un lumen especial. Los catéteres debe ser introducidos por neurocirujanos, con vigilancia estricta de los signos vitales, en una unidad de tratamiento intensivo (UTI) o en pabellón quirúrgico, ya que frecuentemente se trata de pacientes muy graves ${ }^{2.7-5}$. Las complicaciones más comunes del procedimiento son infecciones, hemorragias y obstrucción del catéter ${ }^{6,10,1 !}$.

El objetivo de este trabajo fue describir los resultados de una auditoría sobre la evolución y complicaciones de una serie de pacientes en quienes se realizaron mediciones directas de presión intracraneana.

\section{Pacientes y Método}

Se estudiaron retrospectivamente los antecedentes de 1odos los niños sometidos a vigilancia instrumental conijnua de la presion intracraneana en un período de tres años. entre enero de 1993 y diciembre de 1996 , en la unidad de cuidados intensivos pediátricos (UTIP) de la Clínica Alemana de Santiago. Se registró el diagnóstico. la razón de la indicación de vigilancia el tjpo de catéter empleado, el lugar de inserción de este, la duración de ta medición, las complicaciones del procedimiento y la evolución posterior del paciente. Se consignaron las cifras máximas de presión intracraneana en situaciones basales y sin que mediaran maniobras de aspiración de secreciones, kinesilerapia, tos o estimulación ambiental. Se poso especial atención a la pcesión de perfusión cerebral (PPC) que, como norma, se trata de mantener sobre $50 \mathrm{~mm}$ de $\mathrm{Hg}$. Se consideró que había HIC cuando la PIC era mayor de $15 \mathrm{~mm}$ de $\mathrm{Hg}$. Con mediciones de este orden se aplicaron las medidas antjhipertensivas ${ }^{13}$. En todos los pacientes se bizo tomografía axial computadorizada (TAC) del cerebro antes, durante y después del procedimiento, para pesquisar complicaciones. Las infecciones se rastrearon mediante exámenes citoquinicos y cultivos del LCR, que fueron realizados cada $24 \mathrm{~h}$ a todos los pacientes con catéter intraventricular. Cuando se usaron catéteres intraparen-quiınatosos o subdurales, se vigilaron los signos clínico y de laboratorio sugerentes de infección y se realizaron cultivos de los catéteres despues de retirarlos.

\section{Resultados}

Durante el período evaluado se hicieron mediciones de presión intracraneana en 21 niños, 14 eran varones, el promedio de su edad fue 7 años 1 mes (mediana 7 años), márgenes de 1 mes hasta 15 años. Los diagnósticos de ingreso fueron: tumor cerebral $(n=7)$; TEC por acciden- 
te automovilístico $(n=4)$; lesión por proyectil $(n=1)$; malformacion del SNC $(n=4)$; asfixia por inmersion $(n=3)$ y meningitis bacteriana aguda $(n=2)$. Las razones para indicar la medición fueron: hidrocefalia aguda $(n=13)$, contusión cerebral múltiple y edema cerebral focal $(n=5)$ y edema cerebral difuso $(n=3)$.

En estos pacientes se realizaron 10 intervenciones quirúrgicas para extirpar un tumor cerebral o corregir una malformación del SNC (dos de estos con vaciamiento de hemorragia intracerebral), cuatro para evacuar una hemorragia intracraneana traumática, seis para instalar una válvula de derivación ventrículo-peritoneal. Algunos enfermos fueron sometidos a más de una intervención (ej: extirpación de un tumor e instalación de válvula ventriculoperitoneal). En cinco pacientes se operó sólo para instalar el dispositivo de medición de PIC. En 13 pacientes el dispositivo se puso en el interior de un ventriculo, en 5 en el parénquima y en 3 en posición subdural. Se emplearon catéteres de tibra óptica (E) Camino $\$$ ) en 11 casos, de polietileno en 6 y captador subdural en 4 . El tiempo de inserción del catêter desde el ingreso del niño a la unidad de cuidados intensivos fue menor a 12 horas en 6 pacientes, entre 12 y 24 horas en otros 6 pacientes y mayor a ese plazo en los 9 restantes. La vigilancia instrumental de la presión intracraneana se extendió en promedio por 103 horas. mediana 72 h, márgenes 14 horas a 14 días.

Diecinueve pacientes cursaron con HIC y dos con PIC bajo $15 \mathrm{~mm}$ de $\mathrm{Hg}$ (considerados sin hipertensión intracraneana). En quince enfermos se registraron PIC máximas entre 15 y $40 \mathrm{~mm}$ de $\mathrm{Hg} \mathrm{y}$ en cuatro presiones mayores que 40 $\mathrm{mm}$ de $\mathrm{Hg}$. La mayoría de los pacientes con HIC fueron tratados con varias medidas, incluyendo: hiperventilación para llevar la $\mathrm{PCO} 2$ a cerca de $30 \mathrm{~mm}$ de $\mathrm{Hg}(\mathbf{n}=14)$, drenaje de LCR a través del catéter $(n=13)$, manitol $(n=10)$ y tiopental $(\mathrm{n}=8$ ). En la mayoría de los casos tratados sc usó más de una de estas medidas. En tres casos la presión de perfusion cerebral fue persistentemente inferior a $45 \mathrm{~mm}$ de $\mathrm{Hg}$ pese al tratamiento y todos ellos murieron. En dos de estos pacientes (uno con asfixia por inmersión y otro con TEC y lesiones contuso-hemorrágicas cerebrales) la disminución crítica de la PPC se debió a la elevación de la PIC sobre $40 \mathrm{~mm}$ de $\mathrm{Hg}$, mientras la presión arterial media se mantenfa en los rangos normales o sobre ellos; en el otro, también con asfixia por inmersión, la reducida PPC fue causada por disminución incontrolable de la PAM, pues su presión intracraneal no excedió de $28 \mathrm{~mm}$ de $\mathrm{Hg}$. En un cuarto paciente que sufría por TEC, contusión y hemorragia cerebrales y politraumatismos la PPC fue inferior a $45 \mathrm{~mm}$ de $\mathrm{Hg}$ al comienzo del tratamiento a causa de PAM bajas y PIC moderadamente elevadas, pero luego se recuperó más bien por aumento de la PAM que por disminución significativa de la PIC, pues esta se mantuva en alrededor de $20 \mathrm{~mm}$ de $\mathrm{Hg}$. Este paciente tuvo un curso favorable y fue dado de alta en buenas condiciones. En las lesiones cerebrales difusas o con contusiones localizadas con hipertensión intracraneana ( 7 pacientes), las mayores PIC ocurrieron entre el segundo y el tercer día (promedio 54 horas), pero variando ampliamente entre el primero y quinto día a contar del ingreso.

Se obtuvieron cultivos positivos del LCR en cuatro casos, pero sólo en dos $(9,5 \%$, uno con cada tipo de catéter) se consideró que se trataba de una complicación del procedimiento, debido a que los otros dos sufrían infecciones preexistentes (meningitis bacteriana aguda). Los casos que se consideraron complicaciones ocurrieron en un paciente con una malformación congénita (obstrucción del agujero de Monro) y otro con hidrocefalia por tumor cerebral, en quienes los cultivos dieron resultados positivos al sexto y al séptimo día de instalación del catéter, respectivamente, el que en ambos casos estaba ubicado en el interior de un ventriculo. Los germenes aislados fueron: Staphylococcus aureus (un afectado por malformación congénita), Streptococcus pneumoniae (dos casos: meningitis aguda bacteriana y tumor cerebral) y Streptococcus pyogenes más levaduras (un caso de meningitis aguda bacteriana). La erradicación del germen se logro con la administración de antibióticos intratecales y sistémicos.

Otra complicacion fue la hemorragia intraparenquimatosa registrada en una oportunidad, en un enfermo con meningitis aguda bacteriana en quien el catéter fue instaló como medida de salvataje por hidrocefalia abrupta y signos de enclavamiento de tronco.

Al alta de la unidad, 17 pacientes tenían puntaje normal de Glasgow para el estado de consciencia, y de uno, que sólo tenía 9 puntos al trasladarlo a otro servicio, se desconoce la evolución posterior. Tres de los pacientes con pun- 
taje Glasgow 15 tenían déficit sígnificativas neurologico focal y muscular. Tres pacientes fallecieron, dos con asfixia por inmersión y edema cerebral difuso y uno con TEC grave, contusiones hemorrágicas intracerebrales y traumatismos múltiples, todos ellos incluidos en la descripción de los pacientes con PPC inferiores a $45 \mathrm{~mm}$ de $\mathrm{Hg}$ por $\mathrm{HIC}(\mathrm{n}=2)$ o disminución de la $\operatorname{PAM}(\mathbf{n}=1)$.

\section{Comentario}

En todos los casos, el catéter permitió medir PIC en forma fidedigna según estándares publicados $^{6}$ y decidir medidas terapéuticas precozmente contra la HIC en aquellos casos en que se detectó. En los pacientes en los que no se comprobó, se evitó el uso de medidas innecesarias y potencialmente dañinas, ya que el tratamiento para disminuir la PIC cuando no la hay puede producir isquemia cerebral ${ }^{4}$.

Se confirma en esta serie que la PIC elevada es importante sólo si afecta la PPC y que no necesitan ser extraordinariamente altas para producir daño cerebral si la PAM es coincidentemente baja y ambas determinan baja presión de perfusión. No se ha determinado en niños la cifra ideal de presión de perfusión cerebral, siendo $50 \mathrm{~mm}$ de $\mathrm{Hg}$ una extrapolación de 10 estimado para los adultos. Es lógico suponer que ellas no tengan que ser iguales, como tampoco entre recién nacidos y adolescentes, que para otro de 15 años, como es el caso de esta serie. La duración del déficit de PPC también es importante, como lo muestra el buen resultado en pacientes en que es transitorio.

En los pacientes que evolucionaron con $\mathrm{HIC}_{\text {, }}$ la única expresión de esta fue la medición de la PIC, ante las escasas manifestaciones clínicas en unos y el empleo de medicamentos que las modifican (sedantes, anticonvulsivantes o paralizantes $)^{7.8 .12}$.

La indicación para medir continuamente la presión intracraneana en pacientes con TEC o asfixia por inmersión, se basa en los antecedentes, el examen clínico (escala de Glasgow bajo 8) y la demostración por tomografía axial cerebral de signes de contusión cerebral múltiple en casos de Iraumatismo encefalocraneal o de edema cerebral difuso en los de asfixia por inmersión. Estos pacientes no solo tuvieron las mayores PIC, sino, también, tres de ellos murie- ron, mostrando las relaciones entre el pronóstico y la magnitud de la hipertensión, así como con su respuesta al tratamiento ${ }^{8}$.

La frecuencia de infecciones en esta serie, a pesar de las precauciones, no es desusada ${ }^{2.10}, 11$. Las diferencias de riesgo por tipo de cateter o ubicación de estos no puede ser evaluada en una experiencia tan pequeña, si bien es mayor en posición ventricular ${ }^{10,1]}$. Puesto que el riesgo de infección aumenta con el tiempo, estos dispositivos deben ser retirados en lo posible antes de cinco días, apenas cumplidos los objetivos de su instalación. La infección del dispositivo no influyó en la evolución final de los pacientes de esta serie.

\section{Referencias}

I. McQuillan-KA: Intracranial pressure monitoring: technical imperatives. AACN Clin Issues Crit Care Nurs 1991; 2: 623-638.

2. Voldivia $F$ : Monitoteo de presión istracrajeana. Revista Chilena de Neuro-Psiquiatría 1993; 3I: $299-304$.

3. Valdivia $F$. Saito $G$, Podestó A, et at: Evaluación del sistema de fibra optica para el monitoreo de la presión intracerebral. Revista Chilena de Neurocirugía 1993: 7: 34-39.

4. Miller JD, Dearden NM, Piper IR, Chan KH: Control of intracraneal pressure in patiencs with severe head injury. J Neurotrauma 1992; 9: 317-326.

5. Diez-Lobato R: Presión intracraneal. Fisiopatología. Métodos de medida. En: Casado-Flores J, Serrano A (Eds). Coma en pediatria, Madrid, Ediciones Diaz de Santos S.A. 1997; 91-97.

6. Ghajar $f$ : Intracranial pressure monitoring techniques. New Horizons 1995: 3: 395-399.

7. Unwan $D H$, Giller CA, Kopinik TA: Central nervous system monitoring. What helps, what does not. Surg Clin North Am 1991; 71: 733-747.

8. Frank JI: Large hemispheric infarction, deterioration. and intracranial pressure. Neurology 1995; 45: 12861290.

9. OSullivan MG. Stathum PF, Jones PA. ef al: Role of intracranial pressure monitoring in severely headinjured patients without signs of intracranial hyper. Iension at initial computerized tomography. J Neurosurg 1994; 80 : 46-50.

10. Blei AT, Olafsson S. Webster S. Levy R: Complications of intracranial pressure monitoring in fulminant hepatic failure. Lancet 1993; 34 [: 690-691.

11. Bader IW, Littlejohns L. Pabuer S: Ventriculostomy and intracranial pressure monitoring: In search of a $0 \%$ rate. Heart Lung 1995; 24: 166-172.

12. Irgau I, Koyfinan Y, Tikellis J: Elective intraoperative intracratial pressure monitoring during laparoscopic cholecystectomy. Arch Surg 1995; 130: 1011-1013.

13. Mutoz $G$. Cerda $M$ : Traumatisino encefalocraneano en la infancia. En: Cerda M y Paris E. Cuidados Intensivos en Pedialría. 1a. ed. Santiago, Mediterráneo 1996: 287-294. 